\title{
Muséologies
}

Les cahiers d'études supérieures

\section{Dialogue avec M. André Delisle}

En lien avec l'article Étude des programmes éducatifs offerts au public dans les jardins historiques du Québec

\section{Koliny Chhim}

Volume 1, numéro 2, avril 2007

URI : https://id.erudit.org/iderudit/1033612ar

DOI : https://doi.org/10.7202/1033612ar

Aller au sommaire du numéro

Éditeur(s)

Association Québécoise de Promotion des Recherches Étudiantes en Muséologie (AQPREM)

\section{ISSN}

1718-5181 (imprimé)

1929-7815 (numérique)

Découvrir la revue

Citer ce document

Chhim, K. (2007). Dialogue avec M. André Delisle : en lien avec l'article Étude des programmes éducatifs offerts au public dans les jardins historiques du Québec. Muséologies, 1(2), 122-131. https://doi.org/10.7202/1033612ar d'utilisation que vous pouvez consulter en ligne. 


\section{Dialogue avec}

\section{André Delisle}

En lien avec l'article Étude des programmes éducatifs

offerts au public dans les jardins historiques du Québec

[ réalisé par Koliny Chhim le 6 février 2007]

André Delisle est titulaire d'un baccalauréat général (anthropologie, géographie, histoire et ethnologie), d'un certificat en archéologie, d'une maîtrise en muséologie et d'un diplôme d'études supérieures en gestion des organismes culturels. En 1994, après avoir travaillé dans le milieu de l'archéologie et œuvré pendant quelques années à titre de contractuel en muséologie, André Delisle a été nommé directeur du Musée du Château Ramezay. Au cours des dernières années, il a dirigé un programme de restauration du musée comprenant la restauration du bâtiment, l'aménagement du jardin et la restauration de l'intérieur du château. Il a aussi développé de nombreux partenariats avec des institutions françaises et américaines afin de développer l'expertise en muséologie. 
KOLINY CHHIM

Pourquoi, selon vous, les jardins historiques sontils si peu connus du public et du milieu muséal québécois?

\section{ANDRÉ DELISLE}

Premièrement, parce que la plupart des jardins historiques sont aujourd'hui disparus et qu'ils ont laissé peu de traces. Deuxièmement, je ne suis pas certain que la majorité des muséologues considèrent vraiment que les jardins fassent partie du monde muséal. On les associe souvent àl'aménagement paysager ou encore aux sciences de la nature.
KOLINY CHHIM

Et en quoi les jardins historiques se distinguent-ils des autres types de musées?

\section{ANDRÉ DeLISLE}

Je ne vois pas les jardins historiques spécifiquement comme des musées. Au Musée du Château Ramezay, nous considérons que le jardin est une salle d'exposition extérieure, à la différence que les gens sont moins « captifs" dans les jardins, surtout dans le nôtre parce qu'il est libre d'accès. Il ne s'agit donc pas de la démarche traditionnelle du musée où le visiteur suit un parcours. Notre jardin compte cinq entrées différentes; les visiteurs sont donc libres d'aller et venir

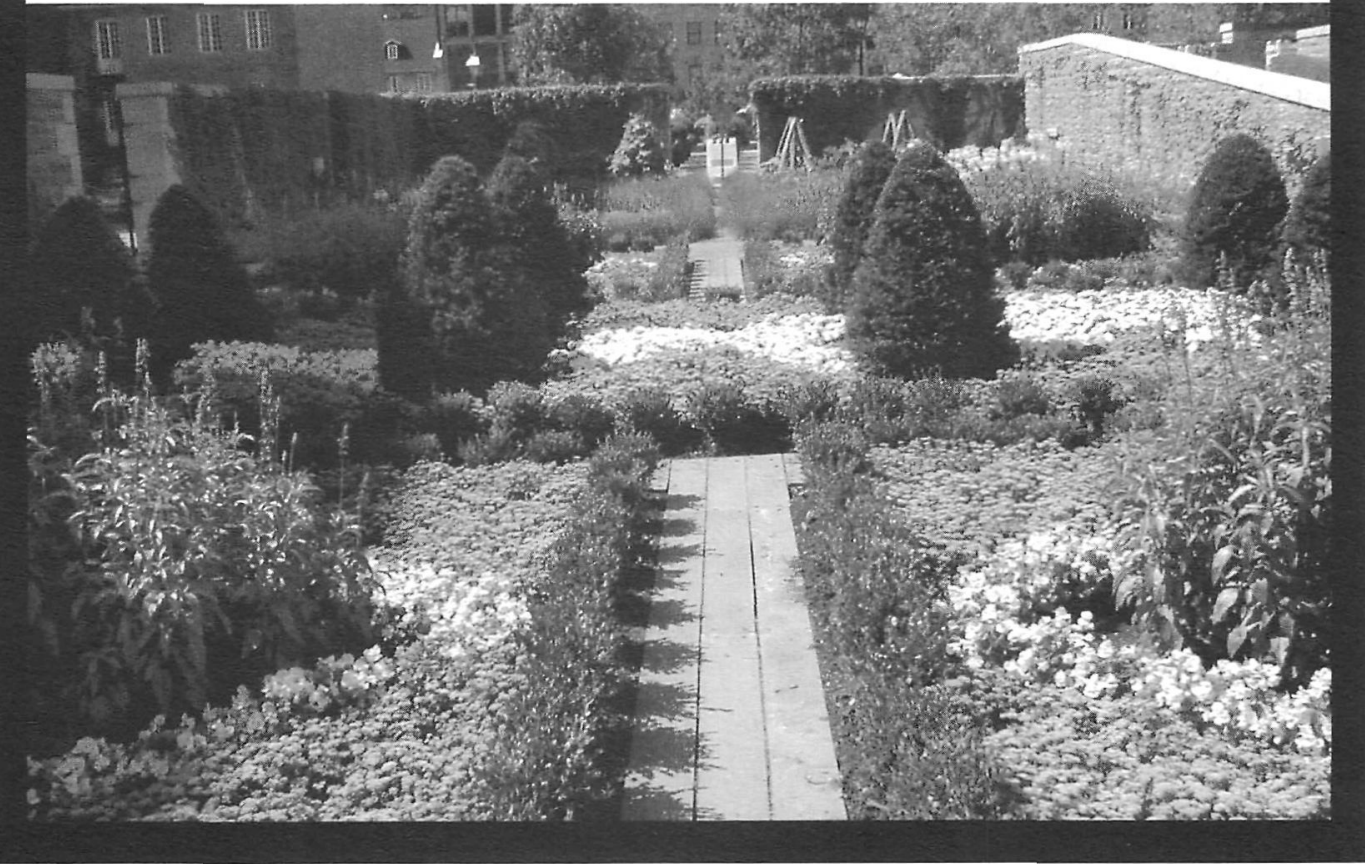


comme ils le souhaitent. Par ailleurs, l'expérience dans un jardin est complètement différente. Tous les sens sont sollicités : la vue, l'ouïe, le toucher, l'odorat et même le goûter parce que, à certaines occasions, les gens sont invités à déguster certaines plantes dans le jardin. C'est une expérience bien différente de celle offerte dans les lieux contemplatifs comme les salles d'exposition.

\section{Koliny ChHIM}

Quel était l'intérêt, pour le Musée du Château Ramezay, de créer un jardin historique ?

\section{ANDRÉ DELISLE}

Ce jardin répondait à deux objectifs. D'abord, la création du jardin faisait partie de notre programme de restauration. Nous souhaitions redonner à l'édifice un environnement qui se rapprochait de ce qu'il était au XVIII' siècle. Le domaine Ramezay était à l'époque beaucoup plus vaste, mais les lots ont été graduellement accaparés et on y a tracé des rues. Avant la construction du jardin, l'endroit était occupé par un stationnement de deux étages. Nous avons récupéré une partie de l'ancien terrain pour redonner un certain cadre au Château Ramezay. Ensuite, c'était évidemment pour prolonger nos espaces à l'extérieur. L'aspect "jardin " était important parce que, au XVIII' siècle, c'était un élément central : la ville de Montréal était couverte de jardins. Tout le monde avait son jardin. Ceux qui n'en avaient pas louaient des espaces pour avoir au moins un potager. Aujourd'hui, on parle très peu de cet aspect de l'histoire, parce que ces jardins sont, pour la plupart, disparus. Ceux qui subsistent, comme celui du séminaire des prêtres de Saint-Sulpice, ne sont ni accessibles, ni aménagés pour l'éducation du public. Nous souhaitions donc créer un jardin à caractère historique qui soit accessible au public.

Koliny ChHim

Justement, ce jardin est une création et non une reconstitution à l'identique d'un site préexistant. Sur quels documents historiques cette création a-t-elle été fondée ? Et quelle place avez-vous accordée à l'interprétation et à l'imaginaire dans l'aménagement du jardin ?

\section{ANDRÉ DELISLE}

Il faut se rappeler qu'au départ le domaine de Ramezay était beaucoup plus vaste. D'une part, étant donnéla contrainte d'espace, il nous était impossible de le recréer tel qu'il était. D'autre part, nous n'avons pas retrouvé de plan précis de son aménagement. La correspondance nous a fourni quelques éléments ici et là, mais ce n'était pas suffisant. Nous avons alors décidé, étant donné notre objectif éducatif, d'utiliser tout l'espace disponible pour illustrer ce que pouvait contenir un jardin au XVIII siècle à Montréal, soit des fleurs, des plantes médicinales, un verger et un potager. Sur le plan de l'aménagement de l'espace, tout a été organisé en carrés symétriques. C'est pour ces raisons que nous décrivons le Jardin du Gouverneur comme étant un jardin dans "l'esprit " de la Nouvelle- 
France. Le contenu a fait l'objet de recherches. Les plantes présentées étaient cultivées ici à l'époque. Toutefois, nous avons fait un peu appel à notre imagination en ce qui concerne l'enceinte du jardin : les jardins du XVIII' siècle étaient entourés de clôtures de pieux, mais, pour conserver une certaine cohérence entre le jardin et la résidence, nous avons plutôt opté pour un mur de pierre. Cela dit, les reconstitutions de jardin sont comme les reconstitutions dans les musées : tout est propre, tout est beau. Je ne suis pas convaincu qu'au XVII' siècle tout était aussi bien entretenu que dans notre jardin! D'ailleurs, si le véritable jardin de Ramezay avait survécu au temps, il aurait évolué, c'est-à-dire qu'un mélange d'eléments du XIX ${ }^{e}$ et du XX $X^{e}$ siècles en ferait certainement partie!

Koliny ChHIM

Est-ce que, selon vous, les jardins historiques conviennent à tous les publics?

\section{ANDRÉ DelisLe}

Je pense que oui, surtout en ce qui concerne le premier niveau. Le Jardin du Gouverneur accueille beaucoup de passants. En ce moment, nous ne possédons pas la technologie pour calculer exactement le nombre de visiteurs, mais nous avons tout de même évalué la fréquentation du jardin quelques jours par mois. En extrapolant nos résultats, nous estimons qu'il $y$ vient plus de cent mille visiteurs par année. On voit bien que ces gens-là ne sont pas nécessairement intéressés par le contenu du musée, parce qu'ils n'y entrent pas. Je pense que les gens apprécient le contact visuel avec l'environnement et l'ambiance du jardin, ce que j'appelle le premier niveau, mais je ne pense pas que tout le monde soit intéressé à en savoir davantage en matière de contenus horticole et historique.

KOLINY CHHIM

Ces visiteurs voient votre jardin plutôt comme un lieu de repos...

ANDRÉ Delisle

Oui, un lieu de détente, de contemplation. Le jardin est joli, les fleurs sont belles... c'est un endroit très apprécié.

Koliny ChHIM

Plus spécifiquement, à quels publics le Jardin du Gouverneur s'adresse-t-il ?

\section{ANDRÉ DELISLE}

Notre principal public est, comme le mentionne Ève-Marie Proulx dans cette même revue, les baby-boomers. C'est la clientèle qui s'intéresse davantage au jardinage et à l'horticulture. En ce qui concerne le public en général, on remarque tout de même, depuis les années 1990, que les gens ont de plus en plus de conscience environnementale et qu'ils sont intéressés à en connaître davantage sur les jardins. Par ailleurs, comme jele disais plus tôt, nous accueillons beaucoup de passants et de touristes, c'est la raison pour laquelle nos panneaux sont en six langues. Malheureusement, si les membres des communautés ethniques sont 
nombreux à se promener dans notre jardin, ils ne viennent pas pour autant visiter notre musée! Il faut dire que l'entrée du jardin est gratuite et que c'est un bel endroit. Les gens sont attirés par le jardin, mais ne se sentent pas interpellés par une histoire qu'ils ne considèrent peut-être pas comme la leur! Nous tentons le plus possible de les mettre à l'aise. Les panneaux en six langues ont étéfaits dans cette optique.

\section{Koliny ChHIM}

Quels moyens de médiation vous semblent les plus appropriés pour diffuser le contenu historique aux différents publics?

\section{ANDRÉ DELISLE}

Je pense qu'il n'y a pas de moyen idéal, parce que la médiation n'est pas une science exacte. Le Jardin du Gouverneur comporte différents moyens de médiation et nous poursuivons le travail de développement. De 1995 à 2000, nous avons fait la recherche. Nous avons travaillé avec différents professionnels : architecte, architecte paysagiste, archéologue, historien, botaniste, horticulteur et autres. Le processus a été trèslong, entre autres parce qu'il fallait d'abord procéder à la démolition du stationnement, ensuite aux fouilles archéologiques et à l'élaboration des plans. À partir de 2000, nous avons abordé la question de la médiation. Nous avons fait des analyses, nous avons observéle comportement des visiteurs et nous les avons questionnés afin de voir comment ils se sentaient par rapport aux jardins, pour déterminer ce qu'ils souhaitaient $y$ voir, $y$ entendre. Ensuite, nous avons conçu des prototypes et les avons mis à l'épreuve pour voir ce que les gens en pensaient. Bien souvent, ce que l'on trouve dans les jardins ce sont les panneaux d'identification des plantes. Ces panneaux, écrits en gros caractères parce qu'ils s'adressent aux baby-boomers, contiennent les noms des plantes en français, en anglais et en latin. Lorsqu'on fait cela systématiquement, on se retrouve dans un jardin de panneaux! Et c'est ce que nous voulions éviter au Jardin $d u$ Gouverneur; nous avons donc identifié certaines plantes par un numéro qui réfere à un guide de visite. Nous avons aussi créé des visites thématiques, mais nous n'offrons pas de visites guidées régulières au jardin. Nous préférons poster une personne costumée danslejardin et que celle-ci entre en contact avec les gens et réponde à leurs questions, sans plus. Par contre, une visite guidée du Vieux-Montréal dont le jardin est le point de départ est offerte. Dans cette visite, à l'aide de plans et d'images, on parle aux gens des jardins montréalais d'autrefois. Mais, pour en revenir spécifiquement au Jardin du Gouverneur, des panneaux sonores très discrets présentent un contenu par niveau : ils traitent du jardin et de ses différentes sections, soit le verger, le jardin ornemental et le potager. Les visiteurs ont aussi la possibilité de se procurer le livret qui contient beaucoup d'information sur les plantes. En ce moment, nous travaillons à la création d'un carnet de jeux pour les enfants. Nous essayons divers moyens 


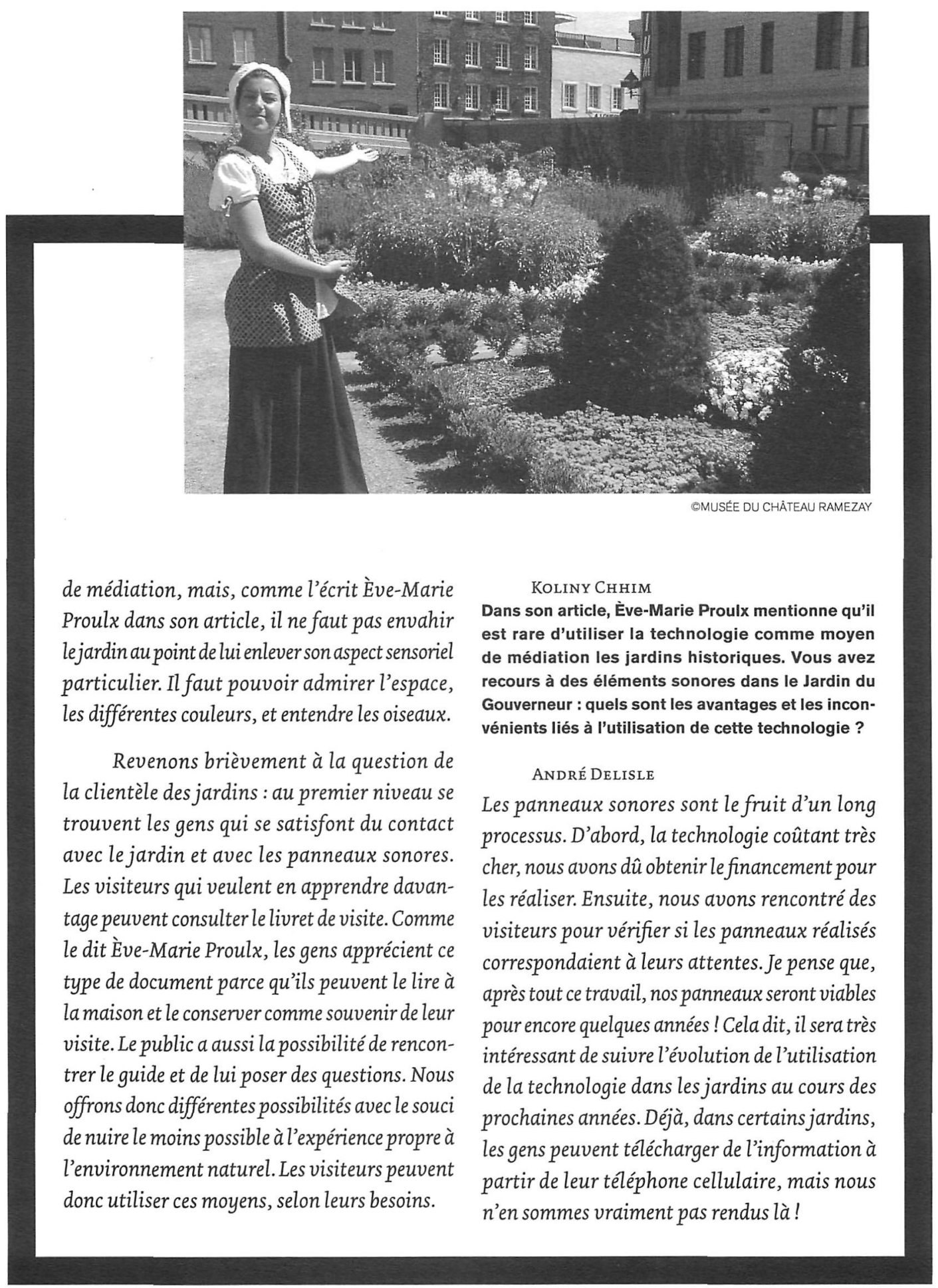




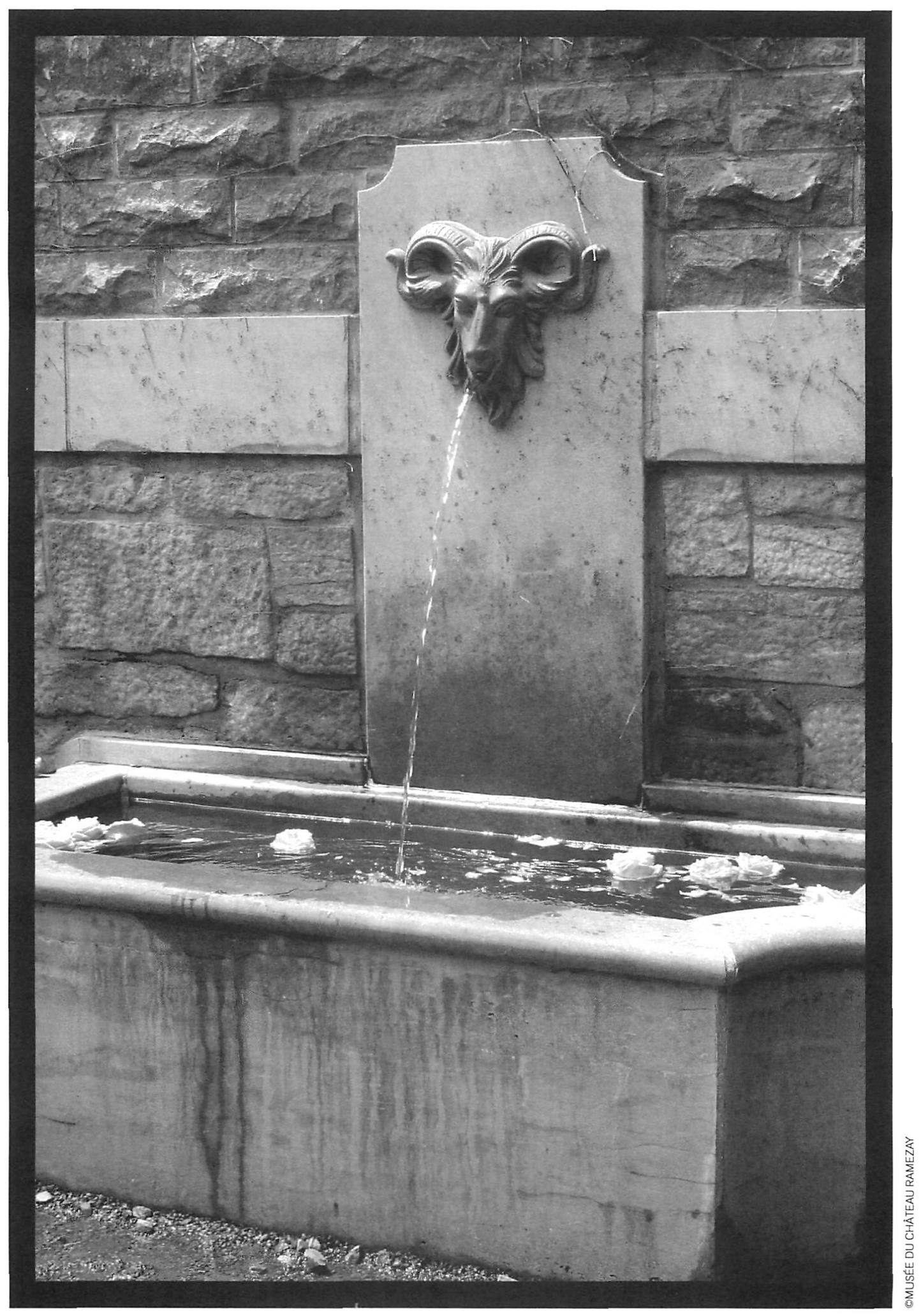


Koliny ChHiM

Quels sont vos commentaires sur cette citation de I'ICOMOS-IFLA (Conseil intemational des monuments et des sites- Fédération internationale des architectes paysagistes) datée de 1971 : " Le jardin ne doit pas être considéré comme un luxe anachronique. [...] II faut susciter la connaissance et la compréhension des jardins historiques et éviter de transformer les jardins historiques en parcs de loisir, les deux fonctions étant inconciliables ${ }^{[1]}$,"

\section{ANDRÉ Delisle}

La première partie de la citation est fantastique. Pour le reste, lorsqu'il est question de parcs de loisir, je pense que c'est peut-être un point de vue européen, parce que là-bas il y a de très vastes jardins propices à l'organisation de concerts et de toutes sortes d'autres activités. Chez nous, c'est moins possible. Je trouve toutefois que le terme " inconciliable " est un peu fort ! J'imagine que l'ICOMOS, à cette époque, essayait de freiner une tendance qui consistait à transformer les grands espaces verts des jardins anciens en salle de concert extérieure.

\section{[1]}

Colloque international sur la conservation et la restauration des jardins historiques, Fontainebleau, France, 13-18 septembre 1971, p. 230.

\section{Koliny ChHiM}

Justement, selon votre expérience, les activités culturelles telles que les pieces de théâtre et les concerts participent-elles réellement à la mise en valeur des jardins historiques?

\section{ANDRÉ DELISLE}

Tout dépend de l'activité...je ne suis pas certain qu'un concert rock à Versailles contribue réellement à la mise en valeur de ses jardins! Cela dit, un concert ou une pièce de théatre en lien avec l'horticulture peut constituer une activité culturelle intéressante. Par exemple, une pièce de théâtre qui raconte l'histoire d'un apothicaire pourrait être présentée dans un jardin. Nous faisons peu d'activités de ce type au jardin, à part l'activité Cortège et sortilèges, en collaboration avec d'autres musées. Il s'agit d'une visite de nuit qui comprend un arrêt au jardin : les visiteurs y aperçoivent une sorcière du XVIII siècle qui cueille des plantes... mais je ne vous en dis pas plus; vous devez venir le découvrir par vous-même! Je pense donc effectivement qu'il est possible d'organiser des activités culturelles qui contribuent à la mission éducative du jardin. 
KOLINY CHHIM

Le jardin se distingue des autres milieux muséaux par son caractère saisonnier. Comment gérez-vous cette situation lorsqu'il est question d'éducation ? Le volet * Jardin " de votre site Internet a-t-il été développé à cet effet ?

\section{ANDRÉ DELISLE}

Effectivement, le caractère saisonnier pose parfois problème. À partir de septembre, il ne reste plus beaucoup de fleurs! Afin de prolonger un peu la période, nous offrons des activités hors saison. Cette année, nous avons élaboré une exposition sur le thème de la courge. Par ailleurs, pendant l'hiver, depuis quelques années, nous présentons des panneaux qui traitent des plantes et de leur vie pendant cette saison. Il est évident quel'hiver la plupart des gensn'iront pas se promener dans le fond du jardin pour lire nos panneaux, mais nous en avons disposé à plusieurs endroits pour rappeler que l'environnement extérieur du chateau est intéressant. Pour contourner le problème des saisons, nous avons aussi intégré le sujet des jardins à nos salles d'expositions permanentes. Par exemple, dans l'exposition Vivre à Montréal au XVIII' siècle, il est question d'alimentation, des produits utilisés et de la manière dont ils étaient cultivés. Finalement, notre site Internet ${ }^{[2]}$ comprend une importante section sur le jardin historique.

[2]

〈http://www.chateauramezay.qc.ca
Toute l'année, les gens peuvent ytrouver une foule de renseignements pour préparer leur visite ou pour en apprendre davantage sur le sujet. Nous parlions de niveaux d'information plus tôt; le site Internet en est un autre. En plus d'offrir du contenu complémentaire, le site permet de présenter le jardin historique à une plus grande échelle : nous pouvons désormais rejoindre des gens partout dans le monde.

\section{Koliny CHнiм}

En terminant, quels seront les défis auxquels les jardins historiques du Québec devront faire face dans les années à venir ? Quels seront les défis du Jardin du Gouverneur?

\section{ANDRÉ DELISLE}

Tout d'abord, il y a la question financière. $A u$ Québec, le financement n'est pas évident. Il n'y a pas d'équivalent des grands jardins français tels Versailles, Fontainebleau ou Villandry. Au Château Ramezay, nous avons la chance de pouvoir compter sur la collaboration de la Ville de Montréal. Sans cet appui, le jardin n'aurait jamais été aussi développé. Par ailleurs, il reste beaucoup de travail à faire, surtout quand on sait que la plupart des Québécois ne connaissent pas l'existence des jardins historiques. Les faire connaître est déjà un beau déf ! Pour contribuer à faire connaître notre jardin et les jardins de Montréal au XVIII' siècle, nous avons choisi de développer différents projets illustrant leur 
importance et leur place dans la vie quotidienne de l'époque. Au cours des dernières années, nous avons présenté plusieurs expositions, parexemple: Aventures botaniques, qui portait surl'exploration, Jardiner à Paris, qui portait sur les jardins de cette ville, et notre exposition sur les courges. Nous concevons des expositions portant sur des aspects spécifiques parce que, sur le plan du jardin lui-même, le contenu est plutôt stable. Nous avons aussi d'autres projets, tels que la réalisation d'une exposition virtuelle et la publication d'ouvrages. Il reste néanmoins que notre plus grand défi concerne l'expertise. Ève-Marie Proulx mentionne qu'il y a peu de publications surlesjardins historiques au Quebec. Effectivement, il $y$ a encore un gros travail de défrichage à faire. Au Château Ramezay, nous avons la chance de réaliser des travaux de recherche à chaque été en collaboration avec l'École du Louvre. Il faut bâtir une expertise dans les sciences connexes au jardin historique parce que, inévitablement, lorsqu'on aborde ce sujet, on parle des plantes, de commerce, des échanges, de l'alimentation, des traditions, de l'environnement et de la vie quotidienne. On parle forcément d'histoire, d'art et de sciences.
Je suis muséologue de formation et je n'ai pas étudié en botanique ou en sciences naturelles. Il en va de même pour les autres employés du Château Ramezay. C'est la raison pour laquelle nous avons créé autant de partenariats. Dans notre cas, c'est vraiment la clé du succès et c'est un défi qui nous occupe régulièrement. Nous travaillons de concert avec des partenaires pour aller chercher les connaissances qui nous manquent : depuis des années, nous collaborons avec les Français qui ont de nombreux jardins dynamiques de taille et, depuis peu, nous travaillons aussi avec les Américains. En ce qui concerne le Chateau Ramezay, ce sont ces différents eléments, soit les partenariats, la recherche, les expositions et les nouvelles activités éducatives, qui contribuent à développer une véritable expertise. Lejardin historique est un lieu extraordinaire de rencontre entre les arts et les sciences. Malheureusement, il est encore perçu comme relevant principalement des sciences naturelles et non de l'histoire. Le pont entre les deux reste à faire. Il est à espérer que, dans un proche avenir, le jardin historique nous permettra de décloisonner ces différents domaines. C'est en tout cas ce que je souhaite! 